\title{
Characterization of Soft Tissue Tumors by Diffusion-Weighted Imaging
}

\author{
Yeliz Pekcevik, ${ }^{1, *}$ Mehmet Onur Kahya, and Ahmet Kaya ${ }^{2}$ \\ ${ }^{1}$ Department of Radiology, Tepecik Training and Research Hospital, Izmir, Turkey \\ ${ }^{2}$ Department of Orthopaedic Surgery, Tepecik Training and Research Hospital, Izmir, Turkey \\ *Corresponding author: Yeliz Pekcevik, Department of Radiology, Tepecik Training and Research Hospital, Izmir, Turkey. Tel:+90-2324696969, Fax: +90-2324330756, E-mail:yelizpekcevik@yahoo.com
}

Received: October 18, 2013; Revised: March 23, 2014; Accepted: July 13, 2014

\begin{abstract}
Background: Diffusion-weighted imaging (DWI) is a noninvasive method for investigation of tumor histological content. It has been applied for some musculoskeletal tumors and reported to be useful.

Objectives: The aim of the present study was to prospectively evaluate the apparent diffusion coefficient (ADC) values of benign and malignant soft tissue tumors and to determine if ADC can help differentiate these tumors.

Patients and Methods: DWI was performed on 25 histologically proven soft tissue masses. It was obtained with a single-shot echo-planar imaging technique using a 1.5T magnetic resonance (MR) machine. The mean ADC values were calculated. We grouped soft tissue tumors as benign cystic, benign solid or mixed, malignant cystic and malignant solid or mixed tumors and compared mean ADC values between these groups.

Results: There was only one patient with a malignant cystic tumor and was not included in the statistical analysis. The median ADC values of benign and malignant tumors were $2.31 \pm 1.29$ and $0.90 \pm 0.70$ (median \pm interquartile range), respectively. The mean ADC values were different between benign and malignant tumors $(\mathrm{P}=0.031)$. Benign cystic tumors had significantly higher ADC values than benign solid or mixed tumors and malignant solid or mixed tumors ( $p$ values were $<0.001$ and 0.003 , respectively). Malignant solid or mixed tumors had lower $\mathrm{ADC}$ values than benign solid or mixed tumors $(\mathrm{P}=0.02)$.

Conclusion: Our preliminary results have shown that although there is some overlap between benign and malignant tumors, adding DWI, MR imaging to routine soft tissue tumor protocols may improve diagnostic accuracy.
\end{abstract}

Keywords: Diffusion Magnetic Resonance Imaging; Soft Tissue Neoplasms; Differential Diagnosis

\section{Background}

Although magnetic resonance imaging (MRI) has an important role in characterization of soft tissue tumors, it lacks specificity for differentiation between benign and malignant lesions (1, 2). Benign lesions are much more common than malignant lesions in daily practice (1). Therefore, we need diagnostic tools that improve our diagnostic confidence and prevent unnecessary biopsies or surgeries.

Diffusion-weighted imaging (DWI) is a noninvasive method for investigation of tumor histological content. Increased apparent diffusion coefficient (ADC) values represent an increase in extracellular water or loss of cell membrane integrity whereas decreased ADC values reflect decrease in extracellular water content or increase in cell number or size (3). These features make DWI an invaluable tool for identifying benign and malignant lesions in the body.

It is generally hard to differentiate between benign and malignant soft tissue tumors from their signal intensities except for some soft tissue tumors that have unique MR imaging signal and localization such as lipoma, ganglion cyst etc. DWI MR has been applied to some soft tissue tumors and reported to be useful $(4,5)$.

\section{Objectives}

The purpose of this study was to prospectively evaluate $\mathrm{ADC}$ values of benign and malignant soft tissue tumors and to determine if ADC values can help differentiate these tumors.

\section{Patients and Methods}

Between May 2012 and March 2013, 25 patients with soft tissue neoplasm (17 males and eight females), aged between 3 and 77 years (mean, 36.6 years) with no previous surgery or treatment were prospectively included in our study. The diagnosis of all masses was confirmed by biopsy and/or surgery after MR imaging.

Nine patients had benign cystic tumors: three had a ganglion cyst, three had complex synovial cyst, two had epidermal cyst and one had complicated Baker's cyst. Ten patients had benign solid or mixed tumors, three had hemangioma, two had schwannoma, while chronic hematoma, neurofibroma (Figure 1), fibromatosis, giant cell tumor of tendon sheath and pigmented villonodular synovitis affected one patient each. There was only one patient in the malignant cystic tumor group (pleomor-

Copyright (C) 2015, Tehran University of Medical Sciences and Iranian Society of Radiology. This is an open-access article distributed under the terms of the Creative Commons Attribution-NonCommercial 4.0 International License (http://creativecommons.org/licenses/by-nc/4.0/) which permits copy and redistribute the material just in noncommercial usages, provided the original work is properly cited. 
PekcevikYetal.

phic liposarcoma). Five patients had malignant solid or mixed tumors, two had soft tissue metastasis from lung adenocarcinoma (Figure 2), one had embryonal rhabdomyosarcoma, one had synovial sarcoma, and one had undifferentiated soft tissue sarcoma. Lesions were located in the thighs (5), knees, ankles (4), buttock, phalanx (2), groin, shoulder, upper arm, forearm and calf (1).

Lipomas were not included in this study because of their classic appearance on conventional MR and the fat-saturated pulse that was used on DWI. In three patients with hemangioma, lesions were intramuscular in location. One patient with chronic hematoma did not have previous trauma or anticoagulant medication history.

The institutional research ethics committee approved this study, and informed consent was obtained from all participants.
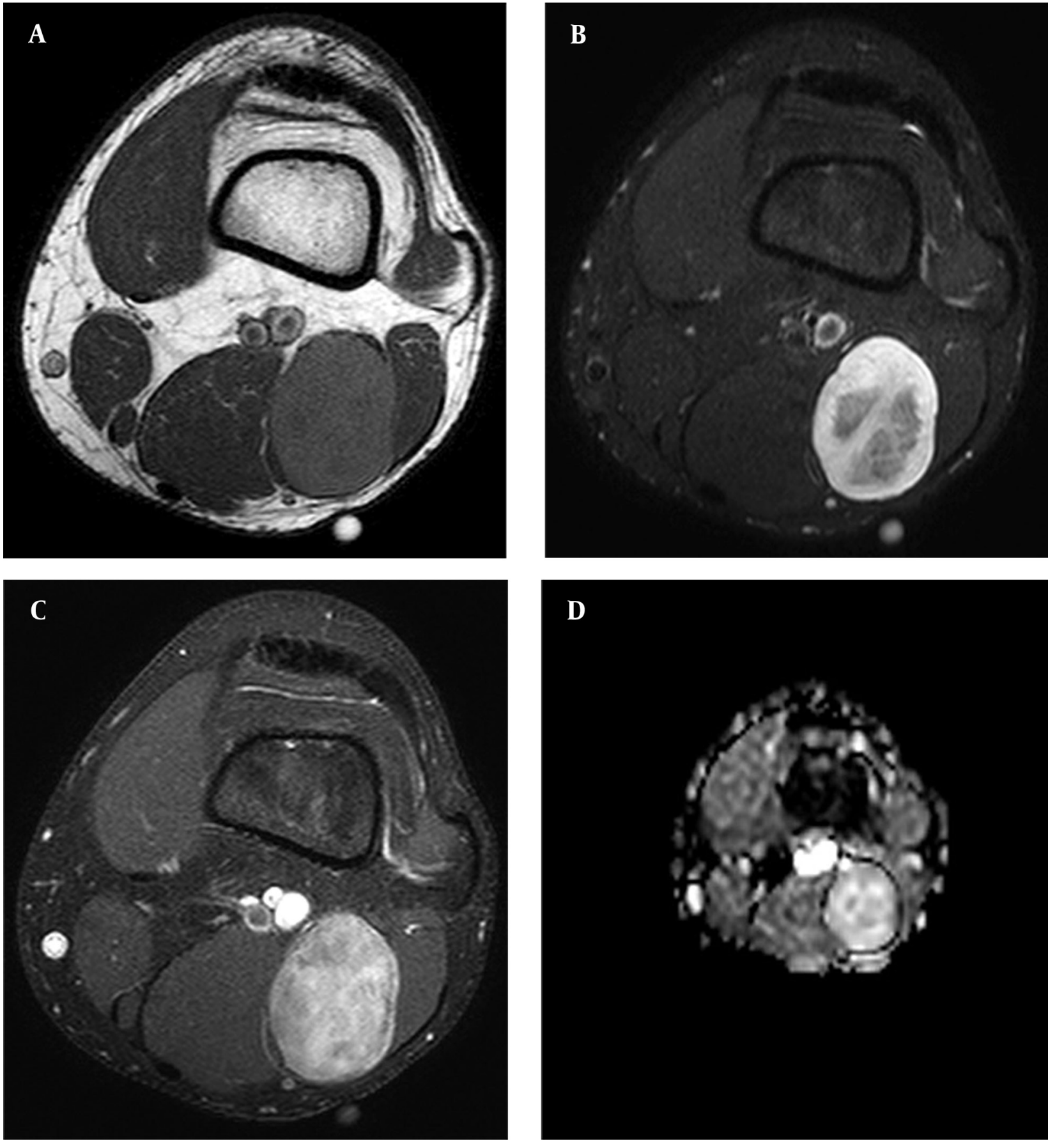

Figure 1. Magnetic resonance imaging of seventeen-year-old male with neurofibroma in the thigh. A, Axial T1-weighted. B, Fat-suppressed proton density. C, T1-weighted fat suppression postcontrast images. D, ADC maps. Mean ADC value was $1.92 \times 10^{-3} \mathrm{~mm}^{2} / \mathrm{sec}$ 
PekcevikYetal.

\subsection{Magnetic Resonance Imaging}

All studies were conducted using a 1.5-T system (Achieva; Philips, The Netherlands). Our routine bone tumor MR imaging protocol includes the following sequences: axial, coronal and/or sagittal T1-weighted, fat-suppressed T2-weighted, dynamic MR imaging and contrast-enhanced fat-suppressed axial, coronal and/or sagittal T1weighted images.

We obtained diffusion-weighted images before con- trast administration for all patients that participated in the study. The pulse sequence used for obtaining the DW images was a single-shot spin-echo Echo-Planar Imaging (EPI) technique with the following parameters: repetition time, $4500 \mathrm{msec}$; echo time, $105 \mathrm{msec}$; directions of the motion-probing gradients, three orthogonal axes; $b$ value, and $1000 \mathrm{sec} / \mathrm{mm}^{2}$; field of view, $220 \mathrm{~mm}$; matrix size, $128 \times 128$; section thickness, $5 \mathrm{~mm}$ with $0.2-1.0-\mathrm{mm}$ intersection gaps; and two signals acquired. Parallel
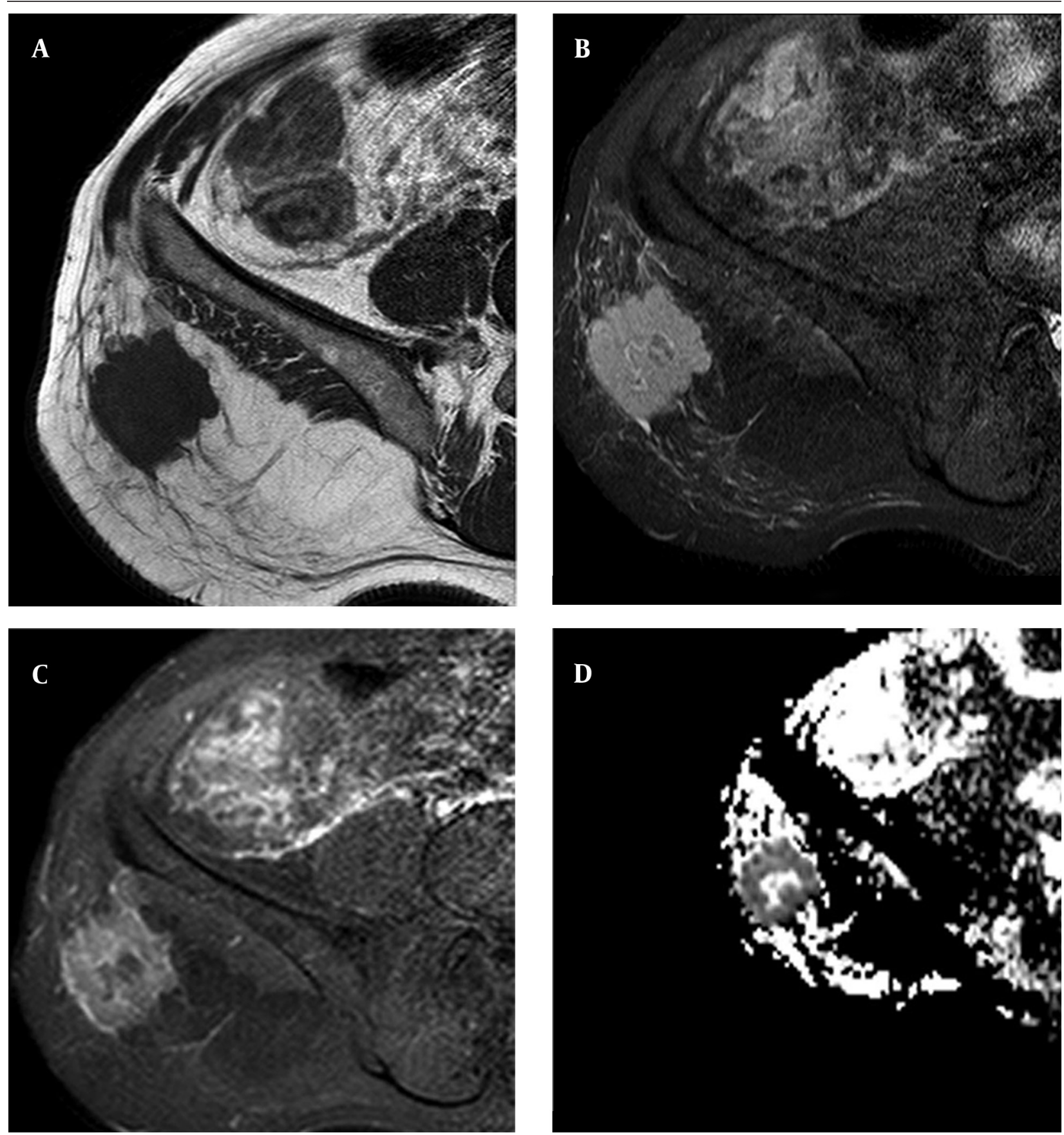

Figure 2. Magnetic resonance imaging of fifty-eight year-old male with subcutaneous fatty tissue metastasis in the right gluteal region from lung adenocarcinoma. A, Sagittal T1-weighted. B, Fat-suppressed proton density. C, T1-weighted fat suppression postcontrast image. D, ADC maps. lung adenocarcinoma, soft tissue metastasis. Mean ADC value from the solid portion was $0.78 \times 10^{-3} \mathrm{~mm}^{2} / \mathrm{sec}$. 
PekcevikYetal.

imaging techniques (SENSE) with a reduction factor of 1 - 1.5 were used. In all images, a fat-saturated pulse was used to exclude chemical-shift artifacts. Three DW images were obtained within an acquisition time of one to two minutes.

ADC maps were automatically generated on the operating console from concurrent images. The ADC values were calculated using the following equation: $A D C=-\ln$ $[S(b) / S(0)] / b$, where $b$ indicates the $b$ value and $S(b)$ and $S(0)$ are the signal intensities of images with $b$ values equal to 1000 and 0 , respectively.

\subsection{Image Analysis}

All the DW images were transferred to a workstation (Easy Vision; Philips Medical Systems). The ADC values were obtained by drawing a region of interest (ROI) on the ADC map and histogram analyses were obtained. The DW image analyses were made by two radiologists independently that were blind to the clinical data of the patients. For solid and mixed tumors, the ROIs were placed on the solid part of the lesions, as selected from T2weighted and contrast enhanced MR images. Cystic and necrotic areas were not included in solid tumors.

\subsection{Statistical Analysis}

We grouped lesions as benign cystic, benign solid or mixed, malignant cystic and malignant solid or mixed tumors. Data analyses were performed using the SPSS software (ver. 15.0, SPSS for windows; SPSS inc., Chicago, IL, USA). The mean ADC values were compared between malignant and benign tumors using the Mann-Whitney U test. We only had one patient with a malignant cystic tumor and did not include it in the statistical analysis. P values of less than 0.05 were considered statistically significant.

\section{Results}

Age, gender, histological diagnosis and mean ADC value of each patients have been mentioned in Table 1 . The median ADC values of benign and malignant tumors were $2.31 \pm 1.29$ and $0.90 \pm 0.70$ (median \pm interquartile range), respectively (Table 2 ). We compared benign and malignant tumors and a significant difference in the ADC values were observed between the two groups $(\mathrm{P}=0.031$ ). Benign cystic tumors had significantly higher ADC values than benign solid or mixed tumors and malignant solid or mixed tumors (P values were $<0.001$ and 0.003 ,

\begin{tabular}{|c|c|c|c|}
\hline Soft Tissue Neoplasm & Age, $y$ & Gender & Mean ADC $\left(\times 10^{-3} \mathrm{~mm}^{2} / \mathrm{sec}\right)$ \\
\hline \multicolumn{4}{|l|}{ Benign cystic tumors } \\
\hline Ganglion cyst & 67 & M & 3.01 \\
\hline Ganglion cyst & 34 & M & 2.99 \\
\hline Ganglion cyst & 40 & M & 2.73 \\
\hline Epidermal cyst & 46 & $\mathrm{~F}$ & 2.57 \\
\hline Epidermal cyst & 17 & M & 2.54 \\
\hline Complicated synovial cyst & 47 & M & 2.42 \\
\hline Complicated synovial cyst & 23 & M & 2.61 \\
\hline Complicated synovial cyst & 50 & M & 2.52 \\
\hline Complicated Baker's cyst & 46 & $\mathrm{~F}$ & 2.76 \\
\hline \multicolumn{4}{|l|}{ Benign solid or mixed tumors } \\
\hline Hemangioma & 28 & M & 2.31 \\
\hline Hemangioma & 22 & $\mathrm{~F}$ & 1.23 \\
\hline Hemangioma & 41 & M & 1.32 \\
\hline Chronic hematoma & 22 & M & 1.24 \\
\hline Schwannoma & 62 & $\mathrm{~F}$ & 1.37 \\
\hline Schwannoma & 45 & $\mathrm{~F}$ & 1.46 \\
\hline Neurofibroma & 17 & M & 1.92 \\
\hline Fibromatosis & 39 & $\mathrm{~F}$ & 1.35 \\
\hline Pigmented villonodular synovitis & 24 & $\mathrm{~F}$ & 0.96 \\
\hline Giant cell tumor of tendon sheath & 42 & M & 0.74 \\
\hline \multicolumn{4}{|l|}{ Malignant cystic tumors } \\
\hline Pleomorphic liposarcoma & 39 & $\mathrm{~F}$ & 2.79 \\
\hline \multicolumn{4}{|l|}{ Malignant solid or mixed tumors } \\
\hline Metastasis (lung, adenocarcinoma) & 52 & M & 1.07 \\
\hline Metastasis (lung, adenocarcinoma) & 58 & M & 0.78 \\
\hline Embryonal rhabdomyosarcoma & 27 & $\mathrm{M}$ & 0.95 \\
\hline Undifferentiated sarcoma & 75 & M & 0.85 \\
\hline Synovial sarcoma & 15 & M & 0.81 \\
\hline
\end{tabular}

\footnotetext{
a Abbreviations: ADC, apparent diffusion coefficient; F, female; M, male.
} 
PekcevikYet al.

\begin{tabular}{|c|c|c|}
\hline Soft Tissue Tumor & Number of Patients, No. (\%) & Median ADC $\left(\times 10^{-3} \mathrm{~mm}^{2} / \mathrm{sec}\right) \pm$ Interquartile Range \\
\hline Benign & $19(76)$ & $2.31 \pm 1.29$ \\
\hline Benign cystic & $9(36)$ & $2.61 \pm 0.35$ \\
\hline Benign solid or mixed & $10(40)$ & $1.34 \pm 0.56$ \\
\hline Malignant & $6(24)$ & $0.90 \pm 0.70$ \\
\hline Malignant cystic & $1(4)$ & - b \\
\hline Malignant solid or mixed & $5(20)$ & $0.85 \pm 0.22$ \\
\hline
\end{tabular}

$\mathrm{b}$ This group only had one patient and was excluded from the statistical analysis.

respectively). Malignant solid or mixed tumors had lower ADC values than benign solid or mixed tumors $(P=0.02)$.

There was some overlap in ADC values between benign and malignant soft tissue tumors. Some benign tumors (giant cell tumor of tendon sheath and fibromatosis) showed very low ADC values. Pleomorphic liposarcoma had ADC values as high as benign cystic tumors.

\section{Discussion}

Our preliminary results showed that although there is some overlap, mean ADC values of benign and malignant soft tissue tumors were statistically different. Adding diffusion-weighted MR to routine soft tissue tumor protocols may improve our diagnostic accuracy. Larger studies with different histological subtypes are required to determine threshold ADC values that can be used in clinical practice.

Differentiating benign and malignant soft tissue tumors in the musculoskeletal system, like other fields in the body, may be possible by DWI. Increased ADC values represent an increase in extracellular water or loss of cell membrane integrity whereas decreased ADC values reflect decrease in extracellular water content or increase in cell number or size $(3,6)$. However, there are some exceptions; malignant tumors usually have lower ADC values and benign tumors usually have higher ADC values. Threshold ADC values for the different tumor types and organs have been reported $(7,8)$. However, to the best of our knowledge, threshold ADC values for soft tissue tumors have not been reported yet $(7,8)$.

We found that the mean ADC values of benign and malignant soft tissue tumors were statistically different. Benign cystic lesions were tumors that had the highest ADC values in our study similar to a previous study by van Rijswijk et al. (4). Giant cell tumors of tendon sheath had the lowest ADC values in our study. Nagata et al. (9) also found low ADC values in giant cell tumors of the tendon sheath and diffuse-type giant cell tumors. Its histologic features, spindle-shaped stromal cells and multinucleated giant cells, are thought to be responsible for low ADC values (10). The diffusion properties of the giant cell tumors of the tendon sheath could be used to differentiate tumor residue or recurrence from postoperative findings $(6,10,11)$.

Most malignant tumors and some benign tumors were heterogeneous in our study. We did our measurement from the solid most homogeneous part of the tumor and did not include cystic or necrotic areas like previous studies (4-6). We used small ROIs for determination of the minimum ADC values of the tumor that reflect the part with highest cellularity $(5,12)$.

Our patient with rhabdomyosarcoma had previous trauma and he was erroneously thought to have chronic hematoma by conventional MRI. We had ADC values (0.95 $\times 10^{-3} \mathrm{~mm}^{2} / \mathrm{sec}$ ) lower than chronic hematoma, similar to a previous study by Oka et al. (5), and this patient was proved to have rhabdomyosarcoma.

We used echo-planar techniques for imaging. The rapid acquisition that reduces artifacts due to patient motion is the major advantage of this technique $(13,14)$. We encountered some magnetic susceptibility artifacts and geometric distortions with EPI yet found it less important than the artifacts caused by movements of the patient.

There were several limitations associated with our study. First, the number of patients, especially those with malignant tumors, was limited in this study. Second, we performed a histogram analysis and used mean ADC values. We did not calculate perfusion effects. However, we analyzed all images with high b value (1000) to reduce contribution of the perfusion effects. Third, the blood products may influence the ADC values of complicated lesions. Last, we did not obtain inter-observer variations of measurement of ADC values in this study.

In conclusion, our preliminary results showed that ADC values are significantly higher in benign tumors. Although there was some overlap between benign and malignant tumors, adding DWI MRI to routine soft tissue tumor protocols may improve diagnostic accuracy. A prospective study of a larger cohort will be required to determine the threshold ADC values that can be used in clinical practice.

\section{Authors' Contributions}

All authors participated equally in this study.

\section{References}

1. Subhawong TK, Wang X, Durand DJ, Jacobs MA, Carrino JA, Machado AJ, et al. Proton MR spectroscopy in metabolic assessment of musculoskeletal lesions. AJR Am J Roentgenol. 2012;198(1):162-72.

2. Gielen JL, De Schepper AM, Vanhoenacker F, Parizel PM, Wang XL, Sciot R, et al. Accuracy of MRI in characterization of soft tissue tu- 
mors and tumor-like lesions. A prospective study in 548 patients. Eur Radiol. 2004;14(12):2320-30.

3. Bley TA, Wieben O, Uhl M. Diffusion-weighted MR imaging in musculoskeletal radiology: applications in trauma, tumors, and inflammation. Magn Reson Imaging Clin N Am. 2009;17(2):263-75.

4. van Rijswijk CS, Kunz P, Hogendoorn PC, Taminiau AH, Doornbos J, Bloem JL. Diffusion-weighted MRI in the characterization of soft-tissue tumors. J Magn Reson Imaging. 2002;15(3):302-7.

5. Oka K, Yakushiji T, Sato H, Yorimitsu S, Hayashida Y, Yamashita Y, et al. Ability of diffusion-weighted imaging for the differential diagnosis between chronic expanding hematomas and malignant soft tissue tumors. J Magn Reson Imaging. 2008;28(5):1195-200.

6. Costa FM, Ferreira EC, Vianna EM. Diffusion-weighted magnetic resonance imaging for the evaluation of musculoskeletal tumors. Magn Reson Imaging Clin N Am. 2011;19(1):159-80.

7. Yamasaki F, Kurisu K, Satoh K, Arita K, Sugiyama K, Ohtaki M, et al. Apparent diffusion coefficient of human brain tumors at MR imaging. Radiology. 2005;235(3):985-91.

8. Noguchi K, Watanabe N, Nagayoshi T, Kanazawa T, Toyoshima S, Shimizu M, et al. Role of diffusion-weighted echo-planar MRI in distinguishing between brain brain abscess and tumour: a preliminary report. Neuroradiology. 1999;41(3):171-4.

9. Nagata S, Nishimura H, Uchida M, Sakoda J, Tonan T, Hiraoka K, et al. Diffusion-weighted imaging of soft tissue tumors: usefulness of the apparent diffusion coefficient for differential diagnosis. Radiat Med. 2008;26(5):287-95.

10. Goldenberg RR, Campbell CJ, Bonfiglio M. Giant-cell tumor of bone. An analysis of two hundred and eighteen cases.J Bone Joint Surg Am. 1970;52(4):619-64.

11. Baur A, Huber A, Arbogast S, Durr HR, Zysk S, Wendtner C, et al. Diffusion-weighted imaging of tumor recurrencies and posttherapeutical soft-tissue changes in humans. Eur Radiol. 2001;11(5):828-33.

12. Oka K, Yakushiji T, Sato H, Hirai T, Yamashita Y, Mizuta H. The value of diffusion-weighted imaging for monitoring the chemotherapeutic response of osteosarcoma: a comparison between average apparent diffusion coefficient and minimum apparent diffusion coefficient. Skeletal Radiol. 2010;39(2):141-6.

13. Hayashida Y, Hirai T, Yakushiji T, Katahira K, Shimomura O, Imuta $\mathrm{M}$, et al. Evaluation of diffusion-weighted imaging for the differential diagnosis of poorly contrast-enhanced and T2-prolonged bone masses: Initial experience. J Magn Reson Imaging. 2006;23(3):377-82.

14. Le Bihan D, Breton E, Lallemand D, Grenier P, Cabanis E, LavalJeantet M. MR imaging of intravoxel incoherent motions: application to diffusion and perfusion in neurologic disorders. Radiology. 1986;161(2):401-7. 